\title{
Narrative review of emerging roles for AKT-mTOR signaling in cancer radioimmunotherapy
}

\author{
Changxian Shen ${ }^{1}$, Yuqi He${ }^{2}$, Qiang Chen ${ }^{3}$, Haihua Feng ${ }^{1}$, Terence M. Williams ${ }^{1}$, Yuanzhi Lu ${ }^{4}$, Zhengfu He \\ ${ }^{1}$ Department of Radiation Oncology, Beckman Research Institute, City of Hope National Medical Center, Duarte, CA, USA; ${ }^{2}$ Monash School of \\ Medicine, Monash University, Clayton, VIC, Australia; ${ }^{3}$ Department of Oncology, The First Affiliated Hospital of Jinan University, Guangzhou, \\ China; ${ }^{4}$ Department of Clinical Pathology, The First Affiliated Hospital of Jinan University, Guangzhou, China; ${ }^{5}$ Department of Thoracic Surgery, \\ Sir Run Run Shaw Hospital, College of Medicine Zhejiang University, Hangzhou, China \\ Contributions: (I) Conception and design: C Shen, TM Williams, Y Lu, Z He; (II) Administrative support: C Shen, Z He; (III) Provision of study \\ materials or patients: C Shen, Z He; (IV) Collection and assembly of data: C Shen, Z He; (V) Data analysis and interpretation: All authors; (VI) \\ Manuscript writing: All authors; (VII) Final approval of manuscript: All authors. \\ Correspondence to: Changxian Shen, PhD. Department of Radiation Oncology, Beckman Research Institute, City of Hope National Medical Center, \\ 1500 E Duarte Road, Duarte, California 91010, USA. Email: cshen@coh.org; Yuanzhi Lu, MD, PhD. Department of Clinical Pathology, The \\ First Affiliated Hospital of Jinan University, 13 Huangpu W Ave, Guangzhou 510630, China. Email: yuanzhi.lu@jnu.edu.cn; Zhengfu He, MD. \\ Department of Thoracic Surgery, Sir Run Run Shaw Hospital, College of Medicine Zhejiang University, East 3 Qingchun Road, Hangzhou 310016 , \\ China. Email: hezhengfu@zju.edu.cn.
}

Objective: To summarize the roles of AKT-mTOR signaling in the regulation of the DNA damage response and PD-L1 expression in cancer cells, and propose a novel strategy of targeting AKT-mTOR signaling in combination with radioimmunotherapy in the era of cancer immunotherapy

Background: Immunotherapy has greatly improved the clinical outcomes of many cancer patients and has changed the landscape of cancer patient management. However, only a small subgroup of cancer patients ( 20-30\%) benefit from immune checkpoint blockade-based immunotherapy. The current challenge is to find biomarkers to predict the response of patients to immunotherapy and strategies to sensitize patients to immunotherapy.

Methods: Search and review the literature which were published in PUBMED from 2000-2021 with the key words mTOR, AKT, drug resistance, DNA damage response, immunotherapy, PD-L1, DNA repair, radioimmunotherapy.

Conclusions: More than $50 \%$ of cancer patients receive radiotherapy during their course of treatment. Radiotherapy has been shown to reduce the growth of locally irradiated tumors as well as metastatic nonirradiated tumors (abscopal effects) by affecting systemic immunity. Consistently, immunotherapy has been demonstrated to enhance radiotherapy with more than one hundred clinical trials of radiation in combination with immunotherapy (radioimmunotherapy) across cancer types. Nevertheless, current available data have shown limited efficacy of trials testing radioimmunotherapy. AKT-mTOR signaling is a major tumor growth-promoting pathway and is upregulated in most cancers. AKT-mTOR signaling is activated by growth factors as well as genotoxic stresses including radiotherapy. Importantly, recent advances have shown that AKT-mTOR is one of the main signaling pathways that regulate DNA damage repair as well as PD-L1 levels in cancers. These recent advances clearly suggest a novel cancer therapy strategy by targeting AKTmTOR signaling in combination with radioimmunotherapy.

Keywords: Mechanistic target of rapamycin (mTOR); AKT; drug resistance; DNA damage response; immunotherapy; programmed death ligand 1 (PD-L1); DNA repair; radioimmunotherapy

Submitted Aug 06, 2021. Accepted for publication Sep 27, 2021.

doi: 10.21037/atm-21-4544

View this article at: https://dx.doi.org/10.21037/atm-21-4544 


\section{Introduction}

There are 20 subfamilies of membrane-bound receptor tyrosine kinases (RTKs), including 58 members (1). These RTKs are important regulators of signal transduction pathways that integrate intracellular and extracellular cues to control cell growth, differentiation, proliferation, survival, and metabolism. Genetic and epigenetic alterations in RTKs result in deregulated kinase activity, leading to changes in multiple downstream signaling pathways (2). Alterations in RTK-mediated signaling pathways are one of the main mechanisms of tumorigenesis and anticancer treatment failure, and targeting RTK signaling is the main strategy for the development of targeted cancer therapy as a monotherapy or in combination with other treatment modalities (2,3). AKT (also called protein kinase B)mechanistic target of rapamycin (mTOR) is one of the most important downstream effectors of RTK signaling $(4,5)$. Deregulation of AKT-mTOR may result from many factors, including, but not limited to, mutations and/or amplification of RTK, overexpression of RTK ligands, mutations and/ or amplification of phosphatidylinositol 3-kinase (PI3K) subunits, and mutations of RAS and phosphatase and tensin homolog (PTEN) (6,7). AKT-mTOR signaling is dysregulated in most cancers and is believed to be an important and attractive cancer therapeutic target. In the past decades, extensive efforts have been made to develop inhibitors targeting AKT-mTOR signaling, particularly mTOR kinase inhibitors. However, though very promising in preclinical studies, the results from most clinical trials are disappointing, with poor effects of these inhibitors as a monotherapy $(8,9)$. To understand the underlying mechanisms by which most cancers are insensitive or not responsive to AKT-mTOR targeted cancer therapy under clinical conditions, there is an urgent need to deeply explore the roles of AKT-mTOR signaling in the regulation of autonomous cancer cells as well as the tumor environment. Recent findings on the essential role of AKTmTOR signaling in regulating cancer immunity (10-13) and the DNA damage response (14-17) may shed light on the distinct discrepancy of the results between preclinical and clinical studies. These recent findings also provide us with new opportunities to rationally combine AKT-mTOR inhibitors with other cancer therapy modalities, particularly immune checkpoint blockade-based immunotherapy. The present review will focus on discussing the mechanisms by which AKT-mTOR signaling regulates programmed death ligand 1 (PD-L1) and the DNA damage response in cancer cells, and proposes the rationale for targeting AKT-mTOR signaling in combination with radiation and immunotherapy. We present the following article in accordance with the Narrative Review reporting checklist (available at https://dx.doi.org/10.21037/atm-21-4544).

\section{AKT-mTOR signaling in cell cycle progression and DNA replication}

Accurate and complete duplication and transmission of DNA to daughter cells is essential for the maintenance and survival of an organism, defects of which lead to human diseases including cancer. AKT-mTOR signaling plays a pivotal role in cell cycle progression by coordinating DNA replication and the activity of cyclin dependent kinases (CDKs), the drivers of cell cycle progression (Figure 1). p21 and p27 are potent and key inhibitors of CDKs (18), and are the key effectors in the control of cell cycle checkpoints by DNA damage checkpoints. Activated AKT phosphorylates p21 and p27 leading to their cytoplasmic accumulation, thereby release and activation of CDKs, especially cyclin D dependent CDK4/6 and cyclin E dependent CDK2 (19-22). In response to DNA damage or replication stress, ATM and ATR checkpoints will be activated to halt cell cycle progression, providing time to repair DNA damage and resolve replication stress (23-25). These are mainly accomplished by $\mathrm{p} 53$ and CHK1, the common downstream effectors of ATM and ATR checkpoints. AKT inhibits the p53-mediated cell cycle checkpoint by phosphorylating and activating MDM2, a direct interactor and inhibitor of the p53 protein. Activation of MDM2 leads to ubiquitination and degradation of $\mathrm{p} 53(26,27)$. CHK1 is the key regulator of both DNA damage and the replication stress response (RSR), and its deregulation results in cell death and genome instability (23). AKT phosphorylates CHK1 at Ser280, which leads to cytoplasmic retention of CHK1, thereby preventing the functions of CHK1 in the nucleus (28). Moreover, translesion DNA synthesis (TLS) is the main mechanism accounting for DNA damage tolerance and the high mutator phenotype of cancer cells. TLS is enhanced by RAD6/RAD18-mediated mono-ubiquitination of proliferating cell nuclear antigen (PCNA), which promotes the switch from replicative DNA polymerases $\delta, \theta$, and $\varepsilon$ to Y-family TLS polymerases $\eta, 1, \kappa$, and Rev 1 (29,30). In response to ultraviolet (UV) radiation, AKT promotes TLS and cell survival by enhancing the mono-ubiquitination of PCNA by RAD6/RAD18 (31). Thus, AKT deregulation may promote cancer cell genome instability and survival 


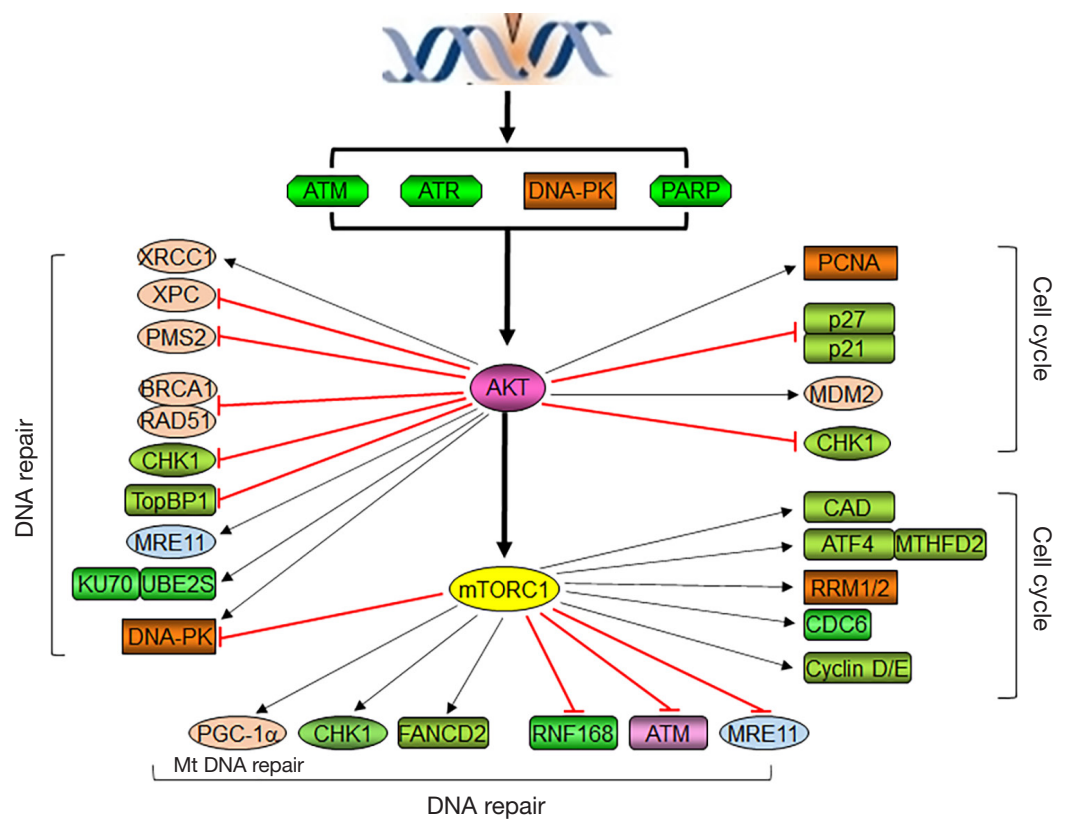

Figure 1 The complex involvement of AKT-mTORC1 signaling in the DNA damage response and cell cycle progression. DNA damaging agent-based chemotherapy and radiotherapy, especially radiotherapy, activate AKT-mTORC1 signaling pathways via ATM, ATR, DNAPK, or PARP depending on the type of DNA damaging agents. Activated AKT kinase regulates the activity of cyclin dependent kinases (CDKs), DNA replication, and DNA repair via multiple mechanisms. mTORC1 is a downstream target of AKT and is the central node in the regulation of DNA replication and DNA repair in response to genotoxic stress in both nuclear and mitochondrial DNA (via PGC-1 $\alpha$ ). The specific mechanisms are detailed in the text.

by enhancing DNA replication under both normal and genotoxic conditions.

The more important mechanisms of AKT in the regulation of DNA replication and cell cycle progression are through its downstream target $\mathrm{mTORC1}$, a protein complex formed by mTOR kinase with mLST8, PRAS40, Deptor, and Raptor. One of the conserved functions of mTORC1 is to promote protein translation via activating p70S6K and eIF4E signaling (32). mTORC1 promotes G1 phase progression and G1/S phase transition through enhancing the protein translation of cyclins D and E, which are the partner and activator of CDK4/6 and CDK2, respectively (33-35). Besides promoting CDK activity, mTORC1 increases DNA replication via multiple other mechanisms. A balanced deoxyribonucleotides (dNTP) pool is essential for accurate and efficient DNA synthesis for replication and repair, defects of which lead to cell death and genomic instability (36-38). mTORC1 increases de novo pyrimidine synthesis via activating p70S6K to phosphorylate CAD (carbamoyl-phosphate synthetase 2, aspartate transcarbamylase, and dihydroorotase) at Ser1859 $(39,40)$ and purine production via the ATF4/MTHFD2 axis (41).
Ribonucleotide reductase (RNR) catalyzes the rate-limiting step in the production of dNTPs from ribonucleotides, and its expression and activity are tightly controlled in all organisms under normal growth and stressful conditions $(36,37,42)$. The mammalian RNR is composed of two identical RRM1 and two small catalytic subunits of either RRM2 or p53R2. Both RRM1 and RRM2 are dynamically regulated during cell cycle progression, while p53R2 is regulated by p53 in response to genotoxic stress (36$38,42)$. The activity of RNR is principally controlled by RRM2 levels in mammalian cells (43). mTOR signaling enhances the cap-dependent protein translation and gene transcription of RRM1 and RRM2, and p53 suppresses RRM1 and RRM2 via inhibiting mTORC1 (44). Similarly, mTOR maintains cell survival but at the cost of an increased mutation rate in response to genotoxins by increasing the expression of RNR subunits in budding yeast (45). Similar regulation of RNR subunit expression by mTOR signaling was found in fission yeast (46). Therefore, mTORC1 upregulation may increase cell survival and accelerate genome instability by enhancing the activities of CDKs and the levels of dNTPs, the elevation of which is also required 
for TLS polymerases to tolerate DNA damage.

Once and only once per cell cycle for each DNA replication is essential for maintaining the integrity of genetic information. A pre-replicative complex (pre$\mathrm{RC}$ ) forms at the origin of replication during late mitosis and early G1 phase (47). In most eukaryotes a pre-RC is composed of six origin recognition complex proteins (ORC1-6), CDC6, CDT1, and a heterohexamer of MCM proteins (MCM2-7). CDC6 and CDT1 are licensing factors of DNA replication and their deregulation results in impaired DNA replication, and therefore, genome instability (48). Insufficient origin licensing during G1 phase, increased and/or ectopic licensing during G1, or re-licensing during the $\mathrm{S}$ and $\mathrm{G} 2$ phases accounts for oncogene-induced replication stress (48-50). Moreover, aberrant expression of CDT1, CDC6, and ORC or abrogation of their regulation results in re-replication of the genome, leading to genome instability (51-53). mTOR signaling has been shown to promote DNA replication origin licensing through upregulating CDC6 $(54,55)$. CDC6 is essential for the loading of the MCM2-7 complex during DNA replication. Consistently, the dramatic reduction of MCM2-7 components and PCNA on chromatin following mTOR inhibition suggests that mTOR may promote the loading and maintenance of the MCM2-7 complex on chromatin by positively regulating CDC6 (54). In addition to replication licensing, CDC6 also plays multiple other roles in ensuring precise chromosome duplication (56), and is crucial for proper S-phase DNA replication progression (57). Moreover, CDC6 can trigger a checkpoint response, which ensures that all DNA is replicated before mitotic entry (58). Taken together, mTOR signaling promotes DNA replication by positively regulating the activity of RNR and the production of purines and pyrimidines, as well as CDC6 expression.

\section{AKT-mTOR signaling in DNA damage response and repair}

All living organisms have developed genome surveillance systems during evolution to cope with constant attacks by physical, chemical, and biological agents (59). A genome surveillance system is a signal transduction cascade composed of signals, sensors, transducers, and effectors (60). ATM-CHK2 and ATR-CHK1 checkpoints surveil the genomic integrity in metazoans. DNA double-stranded breaks (DSBs) produced by ionizing radiation or DNA metabolism-produced reactive oxygen species (ROS) and reactive nitrogen species (RNS) are sensed by the MRE11RAD50-NBS1 complex (MRN), which recruits ATM to damage sites for phosphorylation and activation (61). Activated ATM in turn phosphorylates the MRN complex and histone H2AX to amplify the signals. Following recruitment to DSBs, a plethora of substrates including CHK2, MDM2, and p53 are phosphorylated by ATM with the help of mediators MDC1, 53BP1, and BRCA1. Single-stranded breaks (SSBs) are common DNA damage intermediates produced by different kinds of genotoxins, and are rapidly coated by replicating protein A (RPA) in response to DNA replication fork stalling or slowing down (replication stress). The ATRIP-ATR complex binds to RPA-coated nucleofilament and phosphorylates CHK1 with the help of TOPBP1, Claspin, RAD9-RAD1-HUS1 (9-1-1 complex), and RAD17-RFC clamp loader $(62,63)$. Activated CHK2 and CHK1 phosphorylate numerous downstream effectors to amplify and relay the signals to induce DNA damage responses (DDRs) such as cell cycle arrest, senescence, or apoptosis $(25,59,60)$. ATR-CHK1 is at the heart of the DDR, especially in the RSR $(23,64)$. ATR orchestrates multiple branches of RSR by signaling to arrest cells at the $S$ and G2/M phases, stabilizing stalled replication forks, inhibiting DNA replication of late origin firing, promoting adjacent dormant origin firing and increasing dNTP biosynthesis $(23,25)$. It was reported that transient inhibition of mTOR kinase leads to CHK1 checkpoint activation (54), while long-term mTOR signaling suppression results in decreased CHK1 levels $(65,66)$. In agreement with the essential role of CHK1 in the stabilization of replication forks, mTOR inhibition results in replication fork collapse under DNA lesions and replication stress in yeast (45). Therefore, mTOR signaling maintains cell survival in part by sustaining the CHK1 checkpoint to stabilize replication forks under replication stress.

The Fanconi anemia (FA) signaling pathway maintains genome integrity and cell survival by promoting DNA damage repair through TLS, nucleotide excision repair (NER), and homologous recombination (HR). FA signaling is activated by different kinds of genotoxins and is important for the activation of the ATM-CHK2 and ATR-CHK1 checkpoints. In response to DNA lesions, activation of the FA core E3 ubiquitin ligase complex leads to mono-ubiquitination of FANCI and FANCD2, which are recruited to DNA damage sites to promote DNA repair $(67,68)$. We discovered that FANCD2 is required for timely ATM-CHK2 activation in the early stages of FA 
signaling-mediated repair of interstrand crosslink induced DNA lesions (69). Importantly, we and other labs found that mTOR positively controls FANCD2 expression via multiple mechanisms $(69,70)$. Thus, it is possible that the promotion of FANCD2-dependent activation of the ATM checkpoint in the early response to DNA damage is one of the mechanisms by which AKT-mTOR signaling promotes genome stability under normal growth conditions and cell survival in response to genotoxins. In addition, accumulating evidence has shown an important role for FANCD2 in the maintenance of replication fork stability (71). Under replication stress, mono-ubiquitinated FANCD2 is recruited to stalled replication forks to stabilize forks, restart stalled replication forks, and suppress origin firing (72-74). Taken together, mTOR signaling maintains cell survival and replication fork stability under replication stress through upregulating both CHK1 and FANCD2.

On the other hand, mTORC1 has also been demonstrated to suppress DNA damage response and repair. First, mTORC1 negatively regulates ATM expression in pediatric rhabdomyosarcoma (75). mTORC1 suppresses ATM expression via S6K1/2 signaling by upregulating miR-18a and miR-421, both of which target ATM mRNA. These miRNAs are under the control of the MYCN transcription factor, and one of the mechanisms by which mTORC1 suppresses ATM expression is through sustaining MYCN by S6K1 signaling. Second, mTORC1-S6K signaling can dampen DNA repair via phosphorylation of the E3 ubiquitin ligase RNF168, resulting in its accelerated proteolysis (76). Third, mTORC1 suppresses the activity of DNA-PK through inhibition of PP2A-mediated DNAPK catalytic subunit (DNA-PKcs) dephosphorylation, which is required for DNA-PK activation (77). Moreover, mTORC1 reduces MRE11 protein levels via p70S6K1mediated phosphorylation and degradation of MRE11 (78). Regarding the important roles of ATM and MRE11 in the early stages of DDR, mTORC1 seems to prevent ATM checkpoint activation. Furthermore, RNF168 is recruited to DSB sites to ubiquitinate histone $\mathrm{H} 2 \mathrm{~A}$ at $\mathrm{K} 13 / 15$, which leads to the binding of 53BP1 with H2A-K13/15 to promote NHEJ (79). Therefore, mTORC1 might suppress NHEJ in response to DSBs by inhibiting the functions of DNA-PK and RNF168. However, suppression of the ATM checkpoint and NHEJ by mTORC1 may be cancer type and cell type dependent.

Though upstream of mTORC1 signaling, AKT seems to promote both early DSB repair by regulating DNA-PK and later DSB repair by controlling MRE11. In response to DSBs, AKT binds and promotes the recruitment of DNA-PKcs to DSBs. Moreover, AKT directly phosphorylates DNA-PKcs and increases its activity at DSBs $(80,81)$. Interestingly, AKT physically interacts with and phosphorylates UBE2S at Thr152, leading to the stability and accumulation of UBE2S, which associates with KU70 to enhance NHEJ (82). AKT also increases MRE11 expression after irradiation via the GSK3 $\beta$-catenin/ LEF pathway (83). mTORC1 increases CHK1, while AKT phosphorylates CHK1 leading to CHK1 cytoplasm retention; moreover, AKT phosphorylates TopBP1 (which is required for ATR/CHK1 activation) at S1159 leading to TopBP1 oligomerization, which prevents TopBP1 from interaction with and activation of ATR $(28,84)$. For NER, the role of AKT is controversial, since it can promote the expression of XRCC1 while suppressing the expression of XPC, both of which are the key components of NER machinery $(85,86)$. In addition, dysregulation of DNA mismatch repair (MMR) results in a mutator phenotype and plays an important role in the high tumor mutation burden (TMB) of many cancers. PMS2 is a key component of the MMR machinery and plays an important role in preserving genome stability. It was reported that AKT could modulate PMS2 stability by phosphorylating PMS2, leading to its degradation and impaired nuclear localization $(87,88)$. Moreover, in breast cancer cells, AKT has been shown to inhibit HR by cytoplasmic retention of BRCA1 and RAD51 proteins, resulting in a BRCA1-deficient-like phenotype (89). Overall, AKT seems to promote NHEJ while suppressing HR and MMR, indicating an important role for AKT signaling deregulation in the mutagenesis of cancer cells.

\section{AKT-mTOR signaling in the regulation of PD-L1}

Programmed death-1 (PD-1) and its ligand PD-L1 function as an immune checkpoint that plays a key role in immune homeostasis by fine-tuning the adaptive immune response (90). PD-L1 expression on the cell membrane is tightly controlled at different levels by many factors, including intrinsic and extrinsic factors which modulate constitutive and inducible PD-L1 expression, respectively. For survival, during the multiple processes of tumorigenesis and the evolution of cancer cells, tumor cells gain the capacity to hijack the fine tuning of PD-L1 expression to evade immune responses, thereby escaping death by the host adaptive immune system. Accordingly, targeting PD-1 or PD-L1 with specific inhibitors to prevent their 


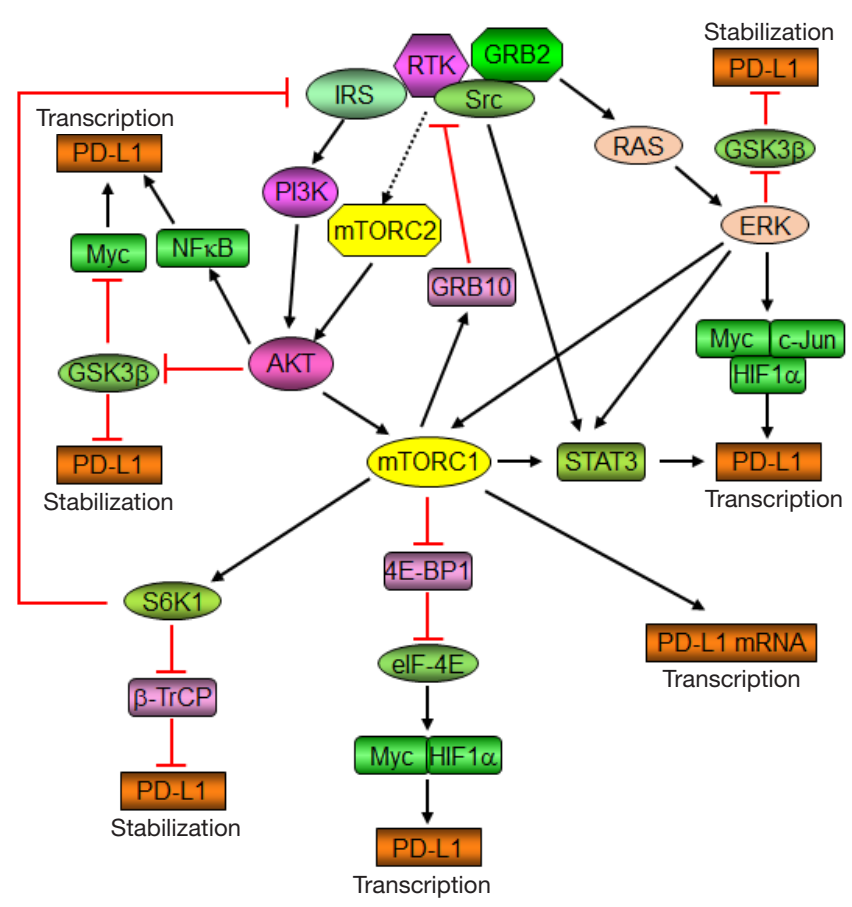

Figure 2 Regulation of PD-L1 by the AKT-mTORC1 signaling pathway. AKT promotes PD-L1 gene transcription via GSK3 $\beta$ myc and $N F-\kappa B$, and $P D-L 1$ protein stabilization via GSK3 $\beta$. mTORC1 increases PD-L1 gene transcription through eIF-4Emediated translation of PD-L1 gene transcription factors myc and HIF1 $\alpha$. It also increases PD-L1 protein stabilization through S6K1- $\beta$ - TrCP and PD-L1 protein translation by promoting the association of PD-L1 mRNA with active polyribosomes. mTORC1 also elevates PD-L1 gene transcription through STAT3, which is activated by RTK and RAS-ERK signaling. RAS-ERK signaling promotes PD-L1 gene transcription via PD-L1 gene transcription factors myc, c-Jun, and HIF1 $\alpha$, and PD-L1 protein stabilization via GSK3 $\beta$. Inhibition of mTORC1 by rapamycin (or rapalogs) results in paradoxical activation of both AKT and ERK signaling via S6K1 and GRB10 negative feedback loops. The specific mechanisms are detailed in the text.

interaction has improved the outcomes of many cancers. Correspondingly, PD-L1 has become a biomarker for cancer immunotherapy, and the detection of PD-L1 by immunohistochemistry using PD-1 or PD-L1 antibodies has become routine in predicting the response of patients to immunotherapy (91-93). Therefore, PD-L1 is both the target and a biomarker for the immunotherapy of many cancers, particularly lung cancers. However, PD-L1 expression on tumor cells is highly variable and is associated with distinct clinicopathological and genomic characteristics in different cancer types, and even the subgroups of the same cancer type (94). Elucidating the mechanisms of PD-L1 deregulation in different caner types with distinct genetic and epigenetic alteration patterns will help to develop effective strategies to delay or overcome the resistance to immunotherapy, which may be achieved by increasing or re-enabling PD-L1 expression on tumor cells or preventing PD-L1 downregulation.

The regulation of PD-L1 expression is wired by complex networks. Extrinsic factors including cytokines, growth factors, hypoxia, chemotherapy, and radiation therapy regulate $\mathrm{PD}-\mathrm{L} 1$ gene transcription. The main intrinsic factors include genetic alterations of the oncogenic signaling pathways RAS/MAPK, PI3K/AKT/mTOR, and JAK/ STAT, tumor suppressors TP53, PTEN, and STK11, DNA damage repair components BRCA1, BRCA2, and ATM, and transcription factors $\mathrm{HIF} 1 \alpha$, c-Myc, and NF- $\mathrm{kB}$. Moreover, CD274 (the gene encodes PD-L1) promoter methylation, inhibition of histone deacetylase, and regulation of gene translation by miRNAs contribute to PD-LI expression at the epigenetic level. Importantly, phosphorylation, ubiquitination, glycosylation, and palmitoylation play an important role in the regulation of PD-L1 protein stability $(12,95)$. How these complex factors are coordinated to tune PD-L1 levels for cancer cells to evade immunity is largely unknown. Answers to this question may be the key to personalized cancer medicine through the combination of immunotherapy with other cancer treatment modalities including radiotherapy, chemotherapy, hormone therapy, and targeted molecular therapies.

AKT-mTOR signaling is the convergence and center of these complicated modules that regulate PD-L1 at the genetic, transcriptional, translational, and post-translational levels (Figure 2). Loss of function mutations of PTEN or gain of function mutations/amplification of PIK3CA lead to activation of AKT-mTOR signaling. Current available data suggests that activated AKT may upregulate $\mathrm{PD}-\mathrm{L} 1$ via several mechanisms (96-98). First, AKT phosphorylates and activates the transcription factor NF- $\mathrm{\kappa B}$ to promote PD-L1 gene transcription (99-101). Second, GSK3 $\beta$ is a key regulator of PD-L1 and directly phosphorylates PD-L1, leading to ubiquitin-proteasome-mediated degradation of PD-L1 (102). GSK3 $\beta$ also phosphorylates and destabilizes c-Myc (103), which is a potent transcription factor for PD-L1 gene transcription (104). AKT may upregulate PD-L1 levels by phosphorylating and inactivating GSK3 $\beta$, which suppresses PD-L1 gene transcription via destabilizing $\mathrm{c}-\mathrm{Myc}$ and promotes $\mathrm{PD}-\mathrm{L} 1$ protein proteolysis. The 
third mechanism of AKT in upregulating $\mathrm{PD}-\mathrm{L} 1$ is through activating its downstream target mTORC1. Both c-Myc and HIF $1 \alpha$ are transcription factors of PD-L1 gene expression $(104,105)$. Activation of mTORC1 by AKT leads to enhanced protein translation of c-Myc and HIF1 $\alpha$ (106), which in turn can promote PD-L1 gene transcription. Moreover, $\beta$-TrCP mediates ubiquitination of PD-L1 to promote its degradation via the proteasome (102), and $\beta$-TrCP activity is inhibited by the direct mTORC1 downstream target p70S6K (107). mTORC1 may increase $\mathrm{PD}-\mathrm{L} 1$ protein accumulation via $\mathrm{p} 70 \mathrm{~S} 6 \mathrm{~K}-\beta-\operatorname{TrCP}$ signaling (107). Moreover, mTORC1 increases the association between PD-L1 mRNA and activated polyribosomes to enhance PD-L1 protein translation (108). In addition, STAT3 is one of the most important transcription factors of PD-L1 gene transcription via IRF1/3 $(108,109)$. mTORC1 phosphorylation and activation of STAT3 may promote its nuclear translocation and activation of PD-L1 gene transcription. Taken together, AKT positively regulates $\mathrm{PD}-\mathrm{L} 1$ at the transcriptional and post-transcriptional levels in mTORC1 dependent and independent ways.

RAS-ERK1/2 signaling is activated by multiple mechanisms including RAS mutation and RTK activation and has a cross talk with mTORC1 signaling. ERK1/2 kinases directly phosphorylate and activate mTORC1, which in turn may increase PD-L1 levels via STAT3, Myc, and HIF1 $\alpha$-mediated PD-L1 gene transcription, p70S6K$\beta$-TrCP induced PD-L1 stabilization, and polyribosome enhanced PD-L1 protein translation (110). Furthermore, through mTORC1, ERK1/2 may directly phosphorylate and activate STAT3 to promote PD-L1 gene transcription (111). Transcription factors c-Jun, Myc, and HIF1 $\alpha$ are important downstream targets of RAS signaling and play key roles in the pathophysiology of human diseases resulting from mutations in RAS-RAF-MEK-ERK signaling $(112,113)$. Independently of mTORC1, activation of ERK1/2 may increase the transcription factors c-Jun, c-Myc, and HIF1 $\alpha$ to promote PD-L1 gene expression (114). In addition, similar to AKT, ERK1/2 may stabilize the PD-L1 protein by directly phosphorylating and inactivating GSK3 $\beta$ (102). Therefore, deregulation of RAS-RAF-MEK-ERK signaling increases PD-L1 levels through mTORC1 dependent and independent mechanisms.

\section{Radiation and immunotherapy}

Targeting immune checkpoint proteins PD-L1/PD-1 and CTLA-4/CD80/CD86 has greatly improved the clinical outcomes of many cancer patients. However, the response of patients with solid tumors to immunotherapy is modest and depends on individual patients (115). Searching for biomarkers to predict the response of cancer patients to immunotherapy and strategies to sensitize tumors to immunotherapy are urgently needed in the field of immunotherapy. Though cancer cells have the same genome as the host body cells, the success of cancer immunotherapy indicates that cancer cells have been "licensed" for immune response. The origin of the "license" of cancer cells for human immunity has been ascribed to cancer genome instability, which produces neoantigens to be recognized by adaptive immunity (116-118). In agreement with this hypothesis, tumors with a higher mutation burden have a better response to immunotherapy (119). Accumulation and tolerance of DNA damage together with impaired DNA damage response and repair contribute to the genome instability of cancer cells $(23,120)$. Therefore, the induction of DNA damage may enhance cancer immunotherapy (121). Conventional radiotherapy and chemotherapy have been attributed to eliciting DNA damage. Emerging evidence has shown that chemoradiation or radiation "boosts" the cancer immunotherapy response $(122,123)$. Radiotherapy has been shown to enhance cancer immunotherapy, leading to more than 100 clinical trials of radiation and immunotherapy combinations across cancer types (124-126). The main rationale of developing cancer radioimmunotherapy is the "boost" of the immune response by radiation via several mechanisms (122). First, due to the intrinsic defects of cancer cells in cell cycle checkpoints, DNA damage checkpoints, DNA replication checkpoints, and mitotic checkpoints (also called spindle assembly checkpoints), irradiated tumor cells with unrepaired and/or incompletely replicated DNA result in cytoplasmic DNA fragments and micronuclei, which activate cGAS/STING signaling. Activation of cGAS/STING signaling induces type I interferons (IFN $\alpha$ and IFN $\beta$ ) to activate the innate immune response (126-129). Second, radiation induces DNA damage leading to neoantigen production, which will be presented to antigen presenting cells (APC) including dendritic cells to activate $\mathrm{T}$ cells in lymph nodes. Activated $\mathrm{T}$ cells will induce the anticancer adaptive immune response both in the locally irradiated tumor cells and non-irradiated metastatic tumors (abscopal effect) (126). Third, radiation treatment of tumor cells with a high mutation burden and microsatellite instability (MSI) increases the production of neoantigens to further enhance the immune response $(125,130)$. In addition, radiation increases $\mathrm{PD}-\mathrm{L} 1$ production through 
the cGAS/STING-IFN $\alpha / \operatorname{IFN} \beta-S T A T 3$ axis as well as activated T cell-mediated IFN $\gamma$-STAT1 signaling $(130,131)$. It seems paradoxical that radiation promotes PD-L1 expression to suppress immunity, however, during immune checkpoint blockade-based immunotherapy, PD-L1 will be neutralized by either anti-PD-1 or PD-L1 antibodies. Most importantly, the induction of PD-L1 expression has been shown to enhance the effects of immunotherapy in many cancers, especially lung cancer (12). Therefore, the net effect of radiotherapy is to enhance immunotherapy by the induction of innate and adaptive anticancer immunity.

\section{Targeting AKT-mTOR signaling in combination with radiation and immunotherapy}

As discussed above, AKT-mTOR signaling upregulates PD-L1 levels (Figure 2) and regulates DNA damage response and repair via multiple mechanisms (Figure 1), which is closely correlated with cancer immunity. Most importantly, genotoxic chemotherapy and radiotherapy induce potent and rapid AKT activation through ATM, ATR, DNA-PK, and PARP depending on the types of DNA lesions (76,132-139). In normal cells, DNA damage/ replication stress activates p53 signaling pathways to inhibit mTORC1 activity by stimulating the expression of TSC2, Sestrins, REDD1, and AMPK $\beta$, all of which are negative regulators of mTORC1 activity. Nevertheless, in most cancer cells, this inhibition of mTORC1 by DNA damage and replication stress has been abolished mainly by TP53 mutations during tumorigenesis. Thus, cancer cells survive genotoxic stresses including DNA damage and replication stress through the activation of ATM/ATR/DNA-PK as well as AKT-mTORC1 signaling.

AKT-mTORC1 is the convergence of RTK and RASERK signaling, which are upregulated in most cancers (32). AKT-mTORC1 signaling is a key regulating module in cell cycle progression and DNA damage repair (140), which has been supported by mounting evidence that molecular targeting of AKT-mTORC1 signaling enhances chemotherapy and radiotherapy-induced DNA damage and replication stress, and sensitizes cancers to chemotherapy and radiotherapy (64,74,140-142). Therefore, the upregulation of AKT-mTORC1 signaling may dampen radiation-produced cytoplasmic DNA fragments and micronuclei to stimulate anticancer innate immunity and neoantigen production to increase anticancer adaptive immunity. In summary, AKT-mTORC1 signaling deregulation leads to the resistance of cancer cells to genotoxin-based chemoradiation and immunotherapy via several mechanisms (Figure 3A). First, AKT-mTORC1 signaling is not only upregulated by genetic and epigenetic alterations but is also activated by chemotherapy and radiotherapy agents. Second, AKT-mTORC1 signaling promotes the survival of autonomous cancer cells by enhancing DNA damage repair. Third, AKT-mTORC1 signaling-mediated DNA damage repair prevents radiation from producing cytoplasmic DNA fragments and micronuclei to stimulate anticancer innate immunity via the cGAS/STING-IFN $\alpha / \mathrm{IFN} \beta$ axis. Fourth, AKT-mTORC1 signaling-mediated DNA damage repair inhibits radiationinduced neoantigen production to increase anticancer adaptive immunity. Most importantly, AKT-mTORC1 signaling elevates PD-L1 levels to enhance immune checkpoints for cancer cells to escape from immune surveillance. Therefore, targeting AKT-mTORC1 signaling may potently sensitize cancer cells to radioimmunotherapy via several mechanisms (Figure $3 B$ ). During cancer radioimmunotherapy, inhibition of AKT-mTORC1 signaling may enhance radiation-produced cytoplasmic DNA fragments and micronuclei to stimulate anticancer innate immunity via the cGAS/STING-IFN $\alpha / \operatorname{IFN} \beta$ axis and neoantigen production to increase anticancer adaptive immunity, while upregulated PD-L1 will be neutralized by antibodies to PD-L1 or PD-1. Thus, the net outcome of AKT-mTORC1 inhibition in combination with radiation and immunotherapy is an enhanced antitumor innate and adaptive immune response.

Intriguingly, though it has been well documented that AKT-mTORC1 signaling positively regulates PD-L1 levels via multiple mechanisms, amounting evidence has demonstrated that targeting AKT-mTORC1 signaling paradoxically leads to PD-L1 upregulation. This is probably due to the complex negative and positive feedback loops of the PI3K-AKT-mTORC1 signaling network (143). For example, inhibition of mTORC1 via either pharmacological inhibition or genetic silencing leads to robust activation of AKT kinase through p70S6K1-IRS1 and GRB10 negative feedback loops $(144,145)$. Moreover, mTORC1 inhibition also induces feedback activation of MAPK/ ERK, which is a key positive regulatory node of PD-L1 $(146,147)$. The paradoxical upregulation of PD-L1 after targeting AKT-mTORC1 signaling may be the main reason for the resistance of cancer cells to inhibitors of AKTmTORC1 signaling in combination with chemotherapy and radiotherapy in various cancer types in clinical trials. This also indicates that the other downstream signaling pathways 

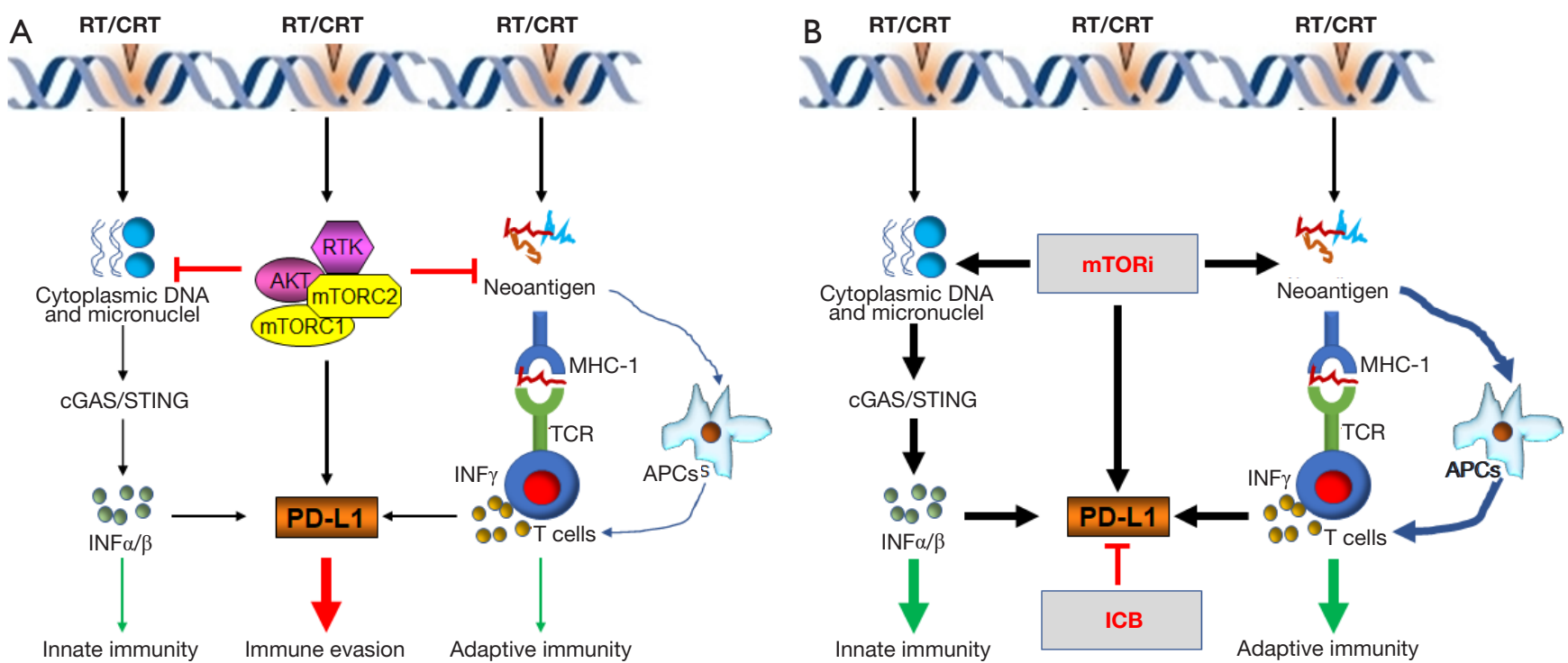

Figure 3 Targeting AKT-mTOR signaling in combination with radioimmunotherapy for cancer treatment. (A) Constitutive activation or radiation therapy (RT)- or chemoradiation therapy (CRT)-induced activation of AKT-mTOR signaling dampens radiation-mediated modulation of innate immunity via preventing the cGAS/STING-IFN $\alpha /$ IFN $\beta$ axis and adaptive immunity by suppressing neoantigen production as well as through increasing PD-1 expression to evade the immune system. (B) Targeting RTK-AKT-mTOR signaling (mTORi) sensitizes cancer cells to radioimmunotherapy. Targeting RTK-AKT-mTOR signaling may enhance innate immunity by promoting the accumulation of cytoplasmic DNA and micronuclei to activate the cGAS/STING-IFN $\alpha / \mathrm{IFN} \beta$ axis and adaptive immunity via elevating neoantigen production to activate $\mathrm{T}$ cells. At the same time, both the enhanced innate and adaptive immunity will increase PD-L1 expression via IFN $\alpha / \mathrm{IFN} \beta$ and IFN $\gamma$, respectively. In addition, targeting RTK-AKT-mTOR signaling may increase PD-L1 levels through the paradoxical reactivation of PI3K-AKT and RAS-ERK signaling (depending on cancer types). However, the effects of increased PD-L1 will be neutralized by anti-PD-1 or PD-L1 antibody immune checkpoint blockade-based immunotherapy (ICB).

of AKT kinases are the major regulators of PD-L1 levels for cancer cells to evade the immune surveillance system. The next challenge is how to rationally target the AKT-mTOR signaling pathways during radioimmunotherapy based on the genomic, epigenetic, transcriptional, post-translational, and metabolic profiles of the tumors from individual cancer patients.

\section{Concluding remarks}

AKT-mTOR signaling is a central integrator and processor of extracellular and intracellular signals. Emerging evidence has shown that AKT-mTOR signaling modulates anticancer immune responses in many clinical trials of combination therapies of AKT-mTOR inhibitors with immune checkpoint inhibitors. Moreover, AKT-mTOR signaling is a key regulator in the supervision of DNA duplication, DNA damage repair, and the replication stress response in the nucleus as well as in mitochondrial DNA repair via PGC-1 $\alpha$ (148). Mounting evidence has demonstrated that immunotherapy enhances radiotherapy, leading to numerous clinical trials of radiation and immunotherapy combinations across cancer types (www. clinicaltrials.gov). Regarding the important roles of AKTmTOR signaling in the regulation of DNA damage repair and PD-L1 expression, it will be promising and attractive to develop preclinical studies and clinical trials targeting AKT-mTOR signaling in combination with radiation and immunotherapy, especially for hard-to-treat cancers such as lung, pancreatic, and brain cancers. Importantly, mutations of PIK3CA and PTEN, which are among the most important regulators of AKT-mTOR signaling, occur at high frequency across cancer types. It will be intriguing to evaluate whether tumors with PIK3CA and PTEN mutations are more sensitive to AKT-mTOR inhibitors in combination with radioimmunotherapy.

\section{Acknowledgments}

Funding: This work was supported by the American 
Cancer Society (IRG-67-003-47), the National Institute of Health (R01CA246553), The Major Research Project of Science Technology Department of Zhejiang Province (2021C03124), and Wu Jieping Medical Foundation (320.6750.19092-12).

\section{Footnote}

Reporting Checklist: The authors have completed the Narrative Review reporting checklist. Available at https:// dx.doi.org/10.21037/atm-21-4544

Conflicts of Interest: All authors have completed the ICMJE uniform disclosure form (available at https://dx.doi. org/10.21037/atm-21-4544). The authors have no conflicts of interest to declare.

Ethical Statement: The authors are accountable for all aspects of the work in ensuring that questions related to the accuracy or integrity of any part of the work are appropriately investigated and resolved.

Open Access Statement: This is an Open Access article distributed in accordance with the Creative Commons Attribution-NonCommercial-NoDerivs 4.0 International License (CC BY-NC-ND 4.0), which permits the noncommercial replication and distribution of the article with the strict proviso that no changes or edits are made and the original work is properly cited (including links to both the formal publication through the relevant DOI and the license). See: https://creativecommons.org/licenses/by-nc-nd/4.0/.

\section{References}

1. Blume-Jensen P, Hunter T. Oncogenic kinase signalling. Nature 2001;411:355-65.

2. Fabbro D. 25 years of small molecular weight kinase inhibitors: potentials and limitations. Mol Pharmacol 2015;87:766-75.

3. Ferguson FM, Gray NS. Kinase inhibitors: the road ahead. Nat Rev Drug Discov 2018;17:353-77.

4. Liu P, Cheng H, Roberts TM, et al. Targeting the phosphoinositide 3-kinase pathway in cancer. Nat Rev Drug Discov 2009;8:627-44.

5. Kim LC, Cook RS, Chen J. mTORC1 and mTORC2 in cancer and the tumor microenvironment. Oncogene 2017;36:2191-201.

6. Liu $\mathrm{W}$, Zhou Y, Reske SN, et al. PTEN mutation: many birds with one stone in tumorigenesis. Anticancer Res 2008;28:3613-9.

7. Mossmann D, Park S, Hall MN. mTOR signalling and cellular metabolism are mutual determinants in cancer. Nat Rev Cancer 2018;18:744-57.

8. Meric-Bernstam F, Gonzalez-Angulo AM. Targeting the mTOR signaling network for cancer therapy. J Clin Oncol 2009;27:2278-87.

9. Paddock MN, Field SJ, Cantley LC. Treating cancer with phosphatidylinositol-3-kinase inhibitors: increasing efficacy and overcoming resistance. J Lipid Res 2019;60:747-52.

10. Sun SY. Searching for the real function of mTOR signaling in the regulation of PD-L1 expression. Transl Oncol 2020;13:100847.

11. Guri Y, Nordmann TM, Roszik J. mTOR at the Transmitting and Receiving Ends in Tumor Immunity. Front Immunol 2018;9:578.

12. Lamberti G, Sisi M, Andrini E, et al. The Mechanisms of PD-L1 Regulation in Non-Small-Cell Lung Cancer (NSCLC): Which Are the Involved Players? Cancers (Basel) 2020;12:3129.

13. Ni JM, Ni AP. Landscape of PD-1/PD-L1 Regulation and Targeted Immunotherapy. Chin Med Sci J 2018;33:174-82.

14. Liu Q, Turner KM, Alfred Yung WK, et al. Role of AKT signaling in DNA repair and clinical response to cancer therapy. Neuro Oncol 2014;16:1313-23.

15. Toulany M, Rodemann HP. Phosphatidylinositol 3-kinase/Akt signaling as a key mediator of tumor cell responsiveness to radiation. Semin Cancer Biol 2015;35:180-90.

16. Ma Y, Vassetzky Y, Dokudovskaya S. mTORC1 pathway in DNA damage response. Biochim Biophys Acta Mol Cell Res 2018;1865:1293-311.

17. Lamm N, Rogers S, Cesare AJ. The mTOR pathway: Implications for DNA replication. Prog Biophys Mol Biol 2019;147:17-25.

18. Otto T, Sicinski P. Cell cycle proteins as promising targets in cancer therapy. Nat Rev Cancer 2017;17:93-115.

19. Hong F, Larrea MD, Doughty C, et al. mTOR-raptor binds and activates SGK1 to regulate p27 phosphorylation. Mol Cell 2008;30:701-11.

20. Medema RH, Kops GJ, Bos JL, et al. AFX-like Forkhead transcription factors mediate cell-cycle regulation by Ras and PKB through p27kip1. Nature 2000;404:782-7.

21. Rössig L, Jadidi AS, Urbich C, et al. Akt-dependent phosphorylation of $\mathrm{p} 21(\mathrm{Cip} 1)$ regulates PCNA binding and proliferation of endothelial cells. Mol Cell Biol 
2001;21:5644-57.

22. Zhou BP, Liao Y, Xia W, et al. Cytoplasmic localization of p21Cip1/WAF1 by Akt-induced phosphorylation in HER2/neu-overexpressing cells. Nat Cell Biol 2001;3:245-52.

23. Saldivar JC, Cortez D, Cimprich KA. The essential kinase ATR: ensuring faithful duplication of a challenging genome. Nat Rev Mol Cell Biol 2017;18:622-36.

24. Lukas J, Lukas C, Bartek J. More than just a focus: The chromatin response to DNA damage and its role in genome integrity maintenance. Nat Cell Biol 2011;13:1161-9.

25. Pilié PG, Tang C, Mills GB, et al. State-of-the-art strategies for targeting the DNA damage response in cancer. Nat Rev Clin Oncol 2019;16:81-104.

26. Mayo LD, Donner DB. A phosphatidylinositol 3-kinase/ Akt pathway promotes translocation of $\mathrm{Mdm} 2$ from the cytoplasm to the nucleus. Proc Natl Acad Sci U S A 2001;98:11598-603.

27. Ogawara Y, Kishishita S, Obata T, et al. Akt enhances Mdm2-mediated ubiquitination and degradation of p53.J Biol Chem 2002;277:21843-50.

28. King FW, Skeen J, Hay N, et al. Inhibition of Chk1 by activated PKB/Akt. Cell Cycle 2004;3:634-7.

29. Hedglin M, Benkovic SJ. Regulation of Rad6/Rad18 Activity During DNA Damage Tolerance. Annu Rev Biophys 2015;44:207-28.

30. Kanao R, Masutani C. Regulation of DNA damage tolerance in mammalian cells by post-translational modifications of PCNA. Mutat Res 2017;803-805:82-8.

31. Villafañez F, García IA, Carbajosa S, et al. AKT inhibition impairs PCNA ubiquitylation and triggers synthetic lethality in homologous recombination-deficient cells submitted to replication stress. Oncogene 2019;38:4310-24.

32. Saxton RA, Sabatini DM. mTOR Signaling in Growth, Metabolism, and Disease. Cell 2017;168:960-76.

33. Averous J, Fonseca BD, Proud CG. Regulation of cyclin D1 expression by mTORC1 signaling requires eukaryotic initiation factor $4 \mathrm{E}$-binding protein 1 . Oncogene 2008;27:1106-13.

34. Fingar DC, Richardson CJ, Tee AR, et al. mTOR controls cell cycle progression through its cell growth effectors S6K1 and 4E-BP1/eukaryotic translation initiation factor 4E. Mol Cell Biol 2004;24:200-16.

35. Oka K, Ohya-Shimada W, Mizuno S, et al. Up-regulation of cyclin-E(1) via proline-mTOR pathway is responsible for HGF-mediated $\mathrm{G}(1) / \mathrm{S}$ progression in the primary culture of rat hepatocytes. Biochem Biophys Res Commun $2013 ; 435: 120-5$
36. Nordlund P, Reichard P. Ribonucleotide reductases. Annu Rev Biochem 2006;75:681-706.

37. Mathews CK. Deoxyribonucleotides as genetic and metabolic regulators. FASEB J 2014;28:3832-40.

38. Kolberg M, Strand KR, Graff P, et al. Structure, function, and mechanism of ribonucleotide reductases. Biochim Biophys Acta 2004;1699:1-34.

39. Ben-Sahra I, Howell JJ, Asara JM, et al. Stimulation of de novo pyrimidine synthesis by growth signaling through mTOR and S6K1. Science 2013;339:1323-8.

40. Robitaille AM, Christen S, Shimobayashi M, et al. Quantitative phosphoproteomics reveal mTORC1 activates de novo pyrimidine synthesis. Science 2013;339:1320-3.

41. Ben-Sahra I, Hoxhaj G, Ricoult SJH, et al. mTORC1 induces purine synthesis through control of the mitochondrial tetrahydrofolate cycle. Science 2016;351:728-33.

42. Aye Y, Li M, Long MJ, et al. Ribonucleotide reductase and cancer: biological mechanisms and targeted therapies. Oncogene 2015;34:2011-21.

43. D'Angiolella V, Donato V, Forrester FM, et al. Cyclin F-mediated degradation of ribonucleotide reductase M2 controls genome integrity and DNA repair. Cell 2012;149:1023-34.

44. He Z, Hu X, Liu W, et al. P53 suppresses ribonucleotide reductase via inhibiting mTORC1. Oncotarget 2017;8:41422-31.

45. Shen C, Lancaster CS, Shi B, et al. TOR signaling is a determinant of cell survival in response to DNA damage. Mol Cell Biol 2007;27:7007-17.

46. Cohen A, Kupiec M, Weisman R. Gad8 Protein Is Found in the Nucleus Where It Interacts with the MluI Cell Cycle Box-binding Factor (MBF) Transcriptional Complex to Regulate the Response to DNA Replication Stress. J Biol Chem 2016;291:9371-81.

47. Masai H, Matsumoto S, You Z, et al. Eukaryotic chromosome DNA replication: where, when, and how? Annu Rev Biochem 2010;79:89-130.

48. Petropoulos M, Champeris Tsaniras S, Taraviras S, et al. Replication Licensing Aberrations, Replication Stress, and Genomic Instability. Trends Biochem Sci 2019;44:752-64.

49. Jones RM, Mortusewicz O, Afzal I, et al. Increased replication initiation and conflicts with transcription underlie Cyclin E-induced replication stress. Oncogene 2013;32:3744-53.

50. Kotsantis P, Silva LM, Irmscher S, et al. Increased global transcription activity as a mechanism of replication stress 
in cancer. Nat Commun 2016;7:13087.

51. Vaziri C, Saxena S, Jeon Y, et al. A p53-dependent checkpoint pathway prevents rereplication. Mol Cell 2003;11:997-1008.

52. Melixetian M, Ballabeni A, Masiero L, et al. Loss of Geminin induces rereplication in the presence of functional p53. J Cell Biol 2004;165:473-82.

53. Klotz-Noack K, McIntosh D, Schurch N, et al. Rereplication induced by geminin depletion occurs from G2 and is enhanced by checkpoint activation. J Cell Sci 2012;125:2436-45.

54. Wu X, Li S, Hu X, et al. mTOR Signaling Upregulates CDC6 via Suppressing miR-3178 and Promotes the Loading of DNA Replication Helicase. Sci Rep 2019;9:9805.

55. Müller D, Shin S, Goullet de Rugy T, et al. eIF4A inhibition circumvents uncontrolled DNA replication mediated by 4E-BP1 loss in pancreatic cancer. JCI Insight 2019;4:121951.

56. Oehlmann M, Score AJ, Blow JJ. The role of Cdc6 in ensuring complete genome licensing and $\mathrm{S}$ phase checkpoint activation. J Cell Biol 2004;165:181-90.

57. Lau E, Zhu C, Abraham RT, et al. The functional role of Cdc6 in S-G2/M in mammalian cells. EMBO Rep 2006;7:425-30.

58. Clay-Farrace L, Pelizon C, Santamaria D, et al. Human replication protein Cdc6 prevents mitosis through a checkpoint mechanism that implicates Chk1. EMBO J 2003;22:704-12.

59. Sancar A, Lindsey-Boltz LA, Unsal-Kaçmaz K, et al. Molecular mechanisms of mammalian DNA repair and the DNA damage checkpoints. Annu Rev Biochem 2004;73:39-85.

60. Kastan MB, Bartek J. Cell-cycle checkpoints and cancer. Nature 2004;432:316-23.

61. Bakkenist CJ, Kastan MB. DNA damage activates ATM through intermolecular autophosphorylation and dimer dissociation. Nature 2003;421:499-506.

62. Wang X, Zou L, Lu T, et al. Rad17 phosphorylation is required for claspin recruitment and Chk1 activation in response to replication stress. Mol Cell 2006;23:331-41.

63. Zou L, Elledge SJ. Sensing DNA damage through ATRIP recognition of RPA-ssDNA complexes. Science 2003;300:1542-8.

64. Lecona E, Fernandez-Capetillo O. Targeting ATR in cancer. Nat Rev Cancer 2018;18:586-95.

65. Zhou X, Liu W, Hu X, et al. Regulation of CHK1 by mTOR contributes to the evasion of DNA damage barrier of cancer cells. Sci Rep 2017;7:1535.

66. Selvarajah J, Elia A, Carroll VA, et al. DNA damageinduced $\mathrm{S}$ and G2/M cell cycle arrest requires mTORC2dependent regulation of Chk1. Oncotarget 2015;6:427-40.

67. Joo W, Xu G, Persky NS, et al. Structure of the FANCIFANCD2 complex: insights into the Fanconi anemia DNA repair pathway. Science 2011;333:312-6.

68. Knipscheer P, Räschle M, Smogorzewska A, et al. The Fanconi anemia pathway promotes replicationdependent DNA interstrand cross-link repair. Science 2009;326:1698-701.

69. Shen C, Oswald D, Phelps D, et al. Regulation of FANCD2 by the mTOR pathway contributes to the resistance of cancer cells to DNA double-strand breaks. Cancer Res 2013;73:3393-401.

70. Guo F, Li J, Du W, et al. mTOR regulates DNA damage response through $\mathrm{NF}-\kappa \mathrm{B}-$ mediated FANCD2 pathway in hematopoietic cells. Leukemia 2013;27:2040-6.

71. Federico MB, Campodónico P, Paviolo NS, et al. Beyond interstrand crosslinks repair: contribution of FANCD2 and other Fanconi Anemia proteins to the replication of DNA. Mutat Res 2018;808:83-92.

72. Chaudhury I, Sareen A, Raghunandan M, et al. FANCD2 regulates BLM complex functions independently of FANCI to promote replication fork recovery. Nucleic Acids Res 2013;41:6444-59.

73. Lossaint G, Larroque M, Ribeyre C, et al. FANCD2 binds MCM proteins and controls replisome function upon activation of s phase checkpoint signaling. Mol Cell 2013;51:678-90.

74. Michl J, Zimmer J, Buffa FM, et al. FANCD2 limits replication stress and genome instability in cells lacking BRCA2. Nat Struct Mol Biol 2016;23:755-7.

75. Shen C, Houghton PJ. The mTOR pathway negatively controls ATM by up-regulating miRNAs. Proc Natl Acad Sci U S A 2013;110:11869-74.

76. Xie X, Hu H, Tong X, et al. The mTOR-S6K pathway links growth signalling to DNA damage response by targeting RNF168. Nat Cell Biol 2018;20:320-31.

77. Li Y, Wang X, Yue $\mathrm{P}$, et al. Protein phosphatase $2 \mathrm{~A}$ and DNA-dependent protein kinase are involved in mediating rapamycin-induced Akt phosphorylation. J Biol Chem 2013;288:13215-24.

78. Piscitello D, Varshney D, Lilla S, et al. AKT overactivation can suppress DNA repair via p70S6 kinase-dependent downregulation of MRE11. Oncogene 2018;37:427-38.

79. Shiloh Y, Ziv Y. The ATM protein kinase: regulating the cellular response to genotoxic stress, and more. Nat Rev 
Mol Cell Biol 2013;14:197-210.

80. Toulany M, Kehlbach R, Florczak U, et al. Targeting of AKT1 enhances radiation toxicity of human tumor cells by inhibiting DNA-PKcs-dependent DNA double-strand break repair. Mol Cancer Ther 2008;7:1772-81.

81. Toulany M, Lee KJ, Fattah KR, et al. Akt promotes post-irradiation survival of human tumor cells through initiation, progression, and termination of DNA-PKcsdependent DNA double-strand break repair. Mol Cancer Res 2012;10:945-57.

82. Hu L, Li X, Liu Q, et al. UBE2S, a novel substrate of Akt1, associates with $\mathrm{Ku} 70$ and regulates DNA repair and glioblastoma multiforme resistance to chemotherapy. Oncogene 2017;36:1145-56.

83. Deng R, Tang J, Ma JG, et al. PKB/Akt promotes DSB repair in cancer cells through upregulating Mre11 expression following ionizing radiation. Oncogene 2011;30:944-55.

84. Liu K, Graves JD, Scott JD, et al. Akt switches TopBP1 function from checkpoint activation to transcriptional regulation through phosphoserine binding-mediated oligomerization. Mol Cell Biol 2013;33:4685-700.

85. Ming M, Shea CR, Guo X, et al. Regulation of global genome nucleotide excision repair by SIRT1 through xeroderma pigmentosum C. Proc Natl Acad Sci U S A 2010;107:22623-8.

86. Toulany M, Dittmann K, Fehrenbacher B, et al. PI3KAkt signaling regulates basal, but MAP-kinase signaling regulates radiation-induced XRCC1 expression in human tumor cells in vitro. DNA Repair (Amst) 2008;7:1746-56.

87. Hinrichsen I, Weßbecher IM, Huhn M, et al. Phosphorylation-dependent signaling controls degradation of DNA mismatch repair protein PMS2. Mol Carcinog 2017;56:2663-8.

88. Jia J, Zhang Y, Cai J, et al. A novel function of protein kinase $\mathrm{B}$ as an inducer of the mismatch repair gene hPMS2 degradation. Cell Signal 2013;25:1498-504.

89. Plo I, Laulier C, Gauthier L, et al. AKT1 inhibits homologous recombination by inducing cytoplasmic retention of BRCA1 and RAD51. Cancer Res 2008;68:9404-12.

90. Xia L, Liu Y, Wang Y. PD-1/PD-L1 Blockade Therapy in Advanced Non-Small-Cell Lung Cancer: Current Status and Future Directions. Oncologist 2019;24:S31-41.

91. Reck M, Rodríguez-Abreu D, Robinson AG, et al. Pembrolizumab versus Chemotherapy for PD-L1Positive Non-Small-Cell Lung Cancer. N Engl J Med 2016;375:1823-33.
92. Mok TSK, Wu YL, Kudaba I, et al. Pembrolizumab versus chemotherapy for previously untreated, PD-L1expressing, locally advanced or metastatic non-small-cell lung cancer (KEYNOTE-042): a randomised, open-label, controlled, phase 3 trial. Lancet 2019;393:1819-30.

93. Aguilar EJ, Ricciuti B, Gainor JF, et al. Outcomes to first-line pembrolizumab in patients with non-small-cell lung cancer and very high PD-L1 expression. Ann Oncol 2019;30:1653-9.

94. Lamberti G, Spurr LF, Li Y, et al. Clinicopathological and genomic correlates of programmed cell death ligand 1 (PD-L1) expression in nonsquamous non-small-cell lung cancer. Ann Oncol 2020;31:807-14.

95. Kobayashi Y, Lim SO, Yamaguchi H. Oncogenic signaling pathways associated with immune evasion and resistance to immune checkpoint inhibitors in cancer. Semin Cancer Biol 2020;65:51-64.

96. Lastwika KJ, Wilson W 3rd, Li QK, et al. Control of PD-L1 Expression by Oncogenic Activation of the AKTmTOR Pathway in Non-Small Cell Lung Cancer. Cancer Res 2016;76:227-38.

97. McGowan M, Hoven AS, Lund-Iversen M, et al. PIK3CA mutations as prognostic factor in squamous cell lung carcinoma. Lung Cancer 2017;103:52-7.

98. Kaneda MM, Messer KS, Ralainirina N, et al. PI3K $\gamma$ is a molecular switch that controls immune suppression. Nature 2016;539:437-42.

99. Gowrishankar K, Gunatilake D, Gallagher SJ, et al. Inducible but not constitutive expression of PD-L1 in human melanoma cells is dependent on activation of NFкB. PLoS One 2015;10:e0123410.

100. Bouillez A, Rajabi H, Jin C, et al. MUC1-C integrates PD-L1 induction with repression of immune effectors in non-small-cell lung cancer. Oncogene 2017;36:4037-46.

101. Guo R, Li Y, Wang Z, et al. Hypoxia-inducible factor-1 $\alpha$ and nuclear factor- $\kappa \mathrm{B}$ play important roles in regulating programmed cell death ligand 1 expression by epidermal growth factor receptor mutants in non-small-cell lung cancer cells. Cancer Sci 2019;110:1665-75.

102. Li CW, Lim SO, Xia W, et al. Glycosylation and stabilization of programmed death ligand-1 suppresses T-cell activity. Nat Commun 2016;7:12632.

103. Sears R, Nuckolls F, Haura E, et al. Multiple Rasdependent phosphorylation pathways regulate Myc protein stability. Genes Dev 2000;14:2501-14.

104. Casey SC, Tong L, Li Y, et al. MYC regulates the antitumor immune response through CD47 and PD-L1. Science 2016;352:227-31. 
105. Noman MZ, Desantis G, Janji B, et al. PD-L1 is a novel direct target of HIF-1, and its blockade under hypoxia enhanced MDSC-mediated T cell activation. J Exp Med 2014;211:781-90.

106. Bjornsti MA, Houghton PJ. Lost in translation: dysregulation of cap-dependent translation and cancer. Cancer Cell 2004;5:519-23.

107.Deng L, Qian G, Zhang S, et al. Inhibition of mTOR complex 1/p70 S6 kinase signaling elevates PD-L1 levels in human cancer cells through enhancing protein stabilization accompanied with enhanced $\beta$-TrCP degradation. Oncogene 2019;38:6270-82 .

108. Sumimoto H, Takano A, Teramoto K, et al. RAS-MitogenActivated Protein Kinase Signal Is Required for Enhanced PD-L1 Expression in Human Lung Cancers. PLoS One 2016;11:e0166626.

109. Garcia-Diaz A, Shin DS, Moreno BH, et al. Interferon Receptor Signaling Pathways Regulating PD-L1 and PDL2 Expression. Cell Rep 2017;19:1189-201.

110. Coelho MA, de Carné Trécesson S, Rana S, et al. Oncogenic RAS Signaling Promotes Tumor Immunoresistance by Stabilizing PD-L1 mRNA. Immunity 2017;47:1083-1099.e6.

111. Nagini S, Sophia J, Mishra R. Glycogen synthase kinases: Moonlighting proteins with theranostic potential in cancer. Semin Cancer Biol 2019;56:25-36.

112. Chang F, Steelman LS, Lee JT, et al. Signal transduction mediated by the Ras/Raf/MEK/ERK pathway from cytokine receptors to transcription factors: potential targeting for therapeutic intervention. Leukemia 2003;17:1263-93.

113. Karnoub AE, Weinberg RA. Ras oncogenes: split personalities. Nat Rev Mol Cell Biol 2008;9:517-31.

114. Koh J, Jang JY, Keam B, et al. EML4-ALK enhances programmed cell death-ligand 1 expression in pulmonary adenocarcinoma via hypoxia-inducible factor (HIF)- $1 \alpha$ and STAT3. Oncoimmunology 2016;5:e1108514.

115. Robert C, Marabelle A, Herrscher H, et al. Immunotherapy discontinuation - how, and when? Data from melanoma as a paradigm. Nat Rev Clin Oncol 2020;17:707-15.

116. Vitale I, Sistigu A, Manic G, et al. Mutational and Antigenic Landscape in Tumor Progression and Cancer Immunotherapy. Trends Cell Biol 2019;29:396-416.

117. Schumacher TN, Schreiber RD. Neoantigens in cancer immunotherapy. Science 2015;348:69-74.

118. Desrichard A, Snyder A, Chan TA. Cancer Neoantigens and Applications for Immunotherapy. Clin Cancer Res
2016;22:807-12.

119. De Mattos-Arruda L, Blanco-Heredia J, Aguilar-Gurrieri $\mathrm{C}$, et al. New emerging targets in cancer immunotherapy: the role of neoantigens. ESMO Open 2020;4:e000684.

120. Goldstein M, Kastan MB. The DNA damage response: implications for tumor responses to radiation and chemotherapy. Annu Rev Med 2015;66:129-43.

121. Weichselbaum RR, Liang H, Deng L, et al. Radiotherapy and immunotherapy: a beneficial liaison? Nat Rev Clin Oncol 2017;14:365-79.

122.Procureur A, Simonaggio A, Bibault JE, et al. Enhance the Immune Checkpoint Inhibitors Efficacy with Radiotherapy Induced Immunogenic Cell Death: A Comprehensive Review and Latest Developments. Cancers (Basel) 2021;13:678.

123. Galluzzi L, Humeau J, Buqué A, et al. Immunostimulation with chemotherapy in the era of immune checkpoint inhibitors. Nat Rev Clin Oncol 2020;17:725-41.

124. Shaverdian N, Lisberg AE, Bornazyan K, et al. Previous radiotherapy and the clinical activity and toxicity of pembrolizumab in the treatment of non-small-cell lung cancer: a secondary analysis of the KEYNOTE-001 phase 1 trial. Lancet Oncol 2017;18:895-903.

125.Lamberti G, Andrini E, Sisi M, et al. Targeting DNA damage response and repair genes to enhance anticancer immunotherapy: rationale and clinical implication. Future Oncol 2020;16:1751-66.

126. Marconi R, Strolin S, Bossi G, et al. A meta-analysis of the abscopal effect in preclinical models: Is the biologically effective dose a relevant physical trigger? PLoS One 2017;12:e0171559.

127. Mouw KW, Goldberg MS, Konstantinopoulos PA, et al. DNA Damage and Repair Biomarkers of Immunotherapy Response. Cancer Discov 2017;7:675-93.

128. Demaria S, Ng B, Devitt ML, et al. Ionizing radiation inhibition of distant untreated tumors (abscopal effect) is immune mediated. Int J Radiat Oncol Biol Phys 2004;58:862-70.

129. Rodriguez-Ruiz ME, Rodriguez I, Garasa S, et al. Abscopal Effects of Radiotherapy Are Enhanced by Combined Immunostimulatory mAbs and Are Dependent on CD8 T Cells and Crosspriming. Cancer Res 2016;76:5994-6005.

130. Ricciuti B, Recondo G, Spurr LF, et al. Impact of DNA Damage Response and Repair (DDR) Gene Mutations on Efficacy of PD-(L)1 Immune Checkpoint Inhibition in Non-Small Cell Lung Cancer. Clin Cancer Res 2020;26:4135-42.

131. Sato H, Niimi A, Yasuhara T, et al. DNA double-strand 
break repair pathway regulates $\mathrm{PD}-\mathrm{L} 1$ expression in cancer cells. Nat Commun 2017;8:1751.

132. Matsuoka S, Ballif BA, Smogorzewska A, et al. ATM and ATR substrate analysis reveals extensive protein networks responsive to DNA damage. Science 2007;316:1160-6.

133. Viniegra JG, Martínez N, Modirassari P, et al. Full activation of $\mathrm{PKB} / \mathrm{Akt}$ in response to insulin or ionizing radiation is mediated through ATM. J Biol Chem 2005;280:4029-36.

134. Caporali S, Levati L, Starace G, et al. AKT is activated in an ataxia-telangiectasia and Rad3-related-dependent manner in response to temozolomide and confers protection against drug-induced cell growth inhibition. Mol Pharmacol 2008;74:173-83.

135. Bozulic L, Surucu B, Hynx D, et al. PKBalpha/Akt1 acts downstream of DNA-PK in the DNA double-strand break response and promotes survival. Mol Cell 2008;30:203-13.

136.Li HF, Kim JS, Waldman T. Radiation-induced Akt activation modulates radioresistance in human glioblastoma cells. Radiat Oncol 2009;4:43.

137.Park J, Feng J, Li Y, et al. DNA-dependent protein kinase-mediated phosphorylation of protein kinase $\mathrm{B}$ requires a specific recognition sequence in the $\mathrm{C}$-terminal hydrophobic motif. J Biol Chem 2009;284:6169-74.

138. Toulany M, Schickfluss TA, Fattah KR, et al. Function of erbB receptors and DNA-PKcs on phosphorylation of cytoplasmic and nuclear Akt at S473 induced by erbB1 ligand and ionizing radiation. Radiother Oncol 2011;101:140-6.

139. Ethier C, Tardif M, Arul L, et al. PARP-1 modulation of mTOR signaling in response to a DNA alkylating agent. PLoS One 2012;7:e47978.

140. Silvera D, Ernlund A, Arju R, et al. mTORC1 and

Cite this article as: Shen C, He Y, Chen Q, Feng H, Williams TM, Lu Y, He Z. Narrative review of emerging roles for AKTmTOR signaling in cancer radioimmunotherapy. Ann Transl Med 2021;9(20):1596. doi: 10.21037/atm-21-4544
-2 Coordinate Transcriptional and Translational Reprogramming in Resistance to DNA Damage and Replicative Stress in Breast Cancer Cells. Mol Cell Biol 2017;37:e00577-16.

141. Mondesire WH, Jian W, Zhang H, et al. Targeting mammalian target of rapamycin synergistically enhances chemotherapy-induced cytotoxicity in breast cancer cells. Clin Cancer Res 2004;10:7031-42.

142. Guri Y, Hall MN. mTOR Signaling Confers Resistance to Targeted Cancer Drugs. Trends Cancer 2016;2:688-97.

143.Xie J, Proud CG. Crosstalk between mTOR complexes. Nat Cell Biol 2013;15:1263-5.

144. Um SH, Frigerio F, Watanabe M, et al. Absence of S6K1 protects against age- and diet-induced obesity while enhancing insulin sensitivity. Nature 2004;431:200-5. Erratum in: Nature 2004;431:485.

145.Hsu PP, Kang SA, Rameseder J, et al. The mTORregulated phosphoproteome reveals a mechanism of mTORC1-mediated inhibition of growth factor signaling. Science 2011;332:1317-22.

146. Carracedo A, Ma L, Teruya-Feldstein J, et al. Inhibition of mTORC1 leads to MAPK pathway activation through a PI3K-dependent feedback loop in human cancer. J Clin Invest 2008;118:3065-74.

147. Wang X, Hawk N, Yue P, et al. Overcoming mTOR inhibition-induced paradoxical activation of survival signaling pathways enhances mTOR inhibitors' anticancer efficacy. Cancer Biol Ther 2008;7:1952-8.

148. Cunningham JT, Rodgers JT, Arlow DH, et al. mTOR controls mitochondrial oxidative function through a YY1-PGC-1alpha transcriptional complex. Nature 2007;450:736-40. 